\section{Case Reports in Dermatology}

\title{
Ulcerated Giant Dermatofibroma following Routine Childhood Vaccination in a Young Boy
}

\author{
Michelle S.Y. Ng Alice Y.W. Foong Mark J.A. Koh \\ Dermatology Service, KK Women's and Children's Hospital, Singapore, Singapore
}

\section{Keywords}

Dermatofibroma - Ulcerated dermatofibroma · Benign fibrous histiocytoma - Immunization . Vaccination · Cutaneous neoplasm

\begin{abstract}
Introduction: The development of cutaneous neoplasms at immunization sites following vaccination is uncommon, and only few have been reported in the literature worldwide. We report an unusual case of an ulcerated giant dermatofibroma that developed as a chronic nonhealing plaque in the immunization scar of a young boy after vaccination. Case Report: A 13-month-old Chinese boy presented with an unusual skin reaction on the vaccination site at the right anterolateral thigh following a routine intramuscular injection of ' 5 -in-1' (diphtheria, tetanus, pertussis, polio and Haemophilus influenzae B) vaccine at 4 months of age. The immunization site developed a slightly raised papule with a central punctum that progressively grew in size, ulcerated and showed occasional bleeding over a span of 9 months. On followup, the lesion showed a chronic granulomatous reaction with surrounding induration and a central scarring. The right inguinal lymph node was palpable. Ultrasound of the lesion showed only nonspecific focal skin thickening. An incisional skin biopsy with careful histopathological evaluation revealed microscopic features consistent with an ulcerated giant dermatofibroma. Conclusion: Neoplastic development in immunization scars following vaccination is a rare occurrence and, hence, makes this case a diagnostic challenge. A high index of suspicion is crucial in atypical presentations of a common skin lesion, as typified by this case. Careful history taking and clinicopathological correlation of clinical findings with gross
\end{abstract}


and microscopic findings along with targeted immunohistological staining is often essential to aid early diagnosis.

\section{Introduction}

Dermatofibroma (DF), also known as superficial benign fibrous histiocytoma, is a common harmless cutaneous lesion that can develop in any patients with or without predisposing factors. Its etiology still remains controversial, and it is not clear whether it results from a true neoplastic process or from a traumatic reaction after a minor trauma, such as an insect bite, a thorn prick or an injection, as seen in $20 \%$ of cases $[1,2]$.

Several cases of intradermal and dermal neoplasms in vaccination scars have been described in the literature $[1,3]$. However, the literature remains limited due to the rarity of $\mathrm{DF}$, with none being reported in the Asia-Pacific context so far. Also, cases of aluminumcontaining adjuvants in vaccines have been reported, triggering off a hypersensitivity reaction to the metallic ion deposited in the skin of patients after vaccination and resulting in a prolonged inflammatory response leading to granulomatous proliferation [4].

We report a rare case of giant DF, which also presented atypically in the form of an atrophic ulcerative lesion following a vaccination injection, and discuss the clinical importance of recognizing some of the possible etiologies in a nonhealing postimmunization wound.

\section{Case Report}

An otherwise healthy 13-month-old Chinese boy developed an unusual skin reaction on the vaccination site at the right thigh. As part of the Singapore National Childhood Immunisation Programme, the child received his routine '5-in-1' (diphtheria, tetanus, pertussis, polio and Haemophilus influenzae B) vaccine injection into his anterolateral right thigh at 4 months of age. The immunization site reaction did not resolve but developed into a papule with a central punctum, which progressively grew in size, ulcerated and showed occasional bleeding over a span of 9 months. The clinical examination of the lesion showed a granulomatous reaction of $2.0 \times 2.3 \mathrm{~cm}$ in size with surrounding induration and a central scarring plaque of $3 \mathrm{~mm}$ (fig. 1). The right inguinal lymph node was palpable. An ultrasound of the lesion showed only nonspecific focal skin thickening. The decision for an incisional skin biopsy was made and unexpectedly showed features of an ulcerated giant DF.

The histological examination demonstrated a thin centrally ulcerated epidermis with a thick overlying fibrino-serous crust mixed with inflammatory cells and red blood cells. There was epidermal hyperplasia with hyperpigmentation of the basal layer. Within the dermis and extending into the superficial subcutaneous fat, there was a poorly circumscribed proliferation of spindle and stellate epithelioid cells arranged in bundles and fascicles. The cells gave round to oval vesicular nuclei, prominent nucleoli and abundant pinkish cytoplasm. Bundles of epithelioid cells surrounding the collagen fibers were visible. No atypia was observed (fig. 2, fig. 3).

PAS and GMS stains were negative for initial suspicion of fungal organisms. ZN stain was also negative for suspected acid-fast bacilli. The cells stained positively for factor XIIIa (fig. 4) and smooth muscle actin immunohistochemistry and stained negatively for S100, desmin, CD68 and CD34 immunohistochemistry. 


\section{Discussion}

DF is a very common cutaneous lesion whose etiology still remains debatable to date, with several theories postulated [5, 6]. DFs occur more commonly in young adulthood, with a slight predominance in females.

There are several subtypes of DFs, namely the cellular, aneurysmal, epithelioid and atypical variants. Here, we discuss a case with the atypical subtype of DF which has rarely been mentioned in the literature and is also uncommon in clinical practice. DFs can present as papular, nodular or even as atrophic plaques, as typified in our case. The atypical subtype with atrophic morphology, as first described by Page and Assaad [2] in 1987, is a rare variant and represents approximately $2 \%$ of all DFs.

\section{Pertinent Clinical Findings}

DFs can develop anywhere on the body surface, but they develop more frequently on the extremities, especially on the lower limbs. They are usually asymptomatic, although pruritus and tenderness can occasionally be present.

Characteristically, they appear as single firm-feeling elevated nodules, although they may occasionally be atrophic and flat, and the color of the lesion varies from cream to yellow-tan and sometimes pale-pink to a darker brown in dark-skinned individuals. A deeper pigmentation may be seen at the periphery of the lesion. The overlying skin may show a characteristic 'dimple sign' if squeezed. The size of the lesion may range from a few millimeters to $2 \mathrm{~cm}$.

Atypical atrophic DFs, however, appear as depressed and/or puckered lesions that represent inward retractions of the skin. The median age of patients is older than in typical DFs. They are smaller in size, measuring about $1.5 \mathrm{~cm}$ in diameter or less, in contrast to the typical papules and nodules that we commonly encounter. They also exhibit a more exaggerated dimpling with lateral pressure [7].

A striking feature in our case is the presence of ulceration on top of the atrophic DF. Though uncommon, previous cases of erosive and ulcerative DFs have been encountered [810]. An important possibility to entertain would be that of a malignant change, although a histological examination has ruled this out as a cause in our patient. We also considered the presence of an ongoing bacterial infection, but cultures turned out to be negative. Given that the location of the lesion was at the lateral aspect of the thigh, it is likely exposed to repetitive microtrauma, and, consequently, an exogenous traumatic etiology was suspected as the most logical explanation of the ulceration of the lesion. Furthermore, histological features consistent with constant irritation, i.e. rubbing and scratching, were evident due to the presence of inflammatory cells as well as surface bleeding.

\section{Pertinent Histological Findings}

In atypical atrophic DFs, histology reveals thinning of the epidermis and dermis, causing a depression of the surface. Dermal atrophy of more than $50 \%$ compared to the adjacent healthy dermis may be the predominant histopathological feature in addition to the usual features of common DF. Like typical DFs, the proliferating cells are also the fibrohistiocytic cells that stain positively for factor XIIIa and negatively for CD34 immunohistochemistry [11]. Our case was typical for the atrophic variant of DF.

Additional stains, such as the Elastica van Gieson stain, could also be added to demonstrate the complete loss of elastin fibers between collagen fibers, which is characteristic of atrophic DFs [11]. 
Making a Diagnosis

The diagnosis of typical DFs is usually straightforward following a clear clinical history and a careful physical examination. An additional pinch test to demonstrate the 'dimple sign' may aid in diagnosis. The use of a dermatoscope is especially helpful and characteristically shows a fine peripheral delicate pigment network with a central scar-like white patch.

In cases of atypical presentation of DFs as in our case, we always need to bear in mind the possibility of sinister mimics, particularly malignant skin neoplasms such as dermatofibrosarcoma, basal cell carcinoma and the desmoplastic form of melanoma, whereby a distinction is only possible with a biopsy and a careful histopathological evaluation with targeted immunohistochemical staining. In case of doubt, a skin biopsy should always be performed.

\section{Vaccination and Occurrence of Neoplasms}

The pathogenesis of neoplasm development following vaccination remains largely unknown, and several theories have been suggested in the literature. In several case reports involving smallpox virus vaccination $[3,12]$, it has been postulated that a normal scar forms following the immunization injection, with the virus replicating itself in the dermis, and a papule appears which soon progresses into a vesicle, then a pustule and eventually a scab, and finally scarring occurs. However, for unknown reasons, if such a replication process becomes uncontrolled, the reaction may lead to a chain of prolonged inflammatory reactions, and this is believed to eventually result in neoplasm formation.

The development of an immunization site into a cutaneous neoplasm is also believed to be secondary to chronic inflammation induced by the vaccine reaction itself [12]. A component of the vaccine may cause prolonged inflammation as a result of a hypersensitivity reaction, and studies have shown that vaccine adjuvants, such as aluminum, are a major factor for carcinogenesis [4].

The route of administration may also contribute to carcinogenesis [12]. Vaccines that need to be injected intradermally or deeper, for example intramuscularly as in our case, can introduce keratin into the dermal layer along with the needle during the injection and cause a subsequent granulomatous inflammatory reaction in the dermis. Chronic inflammation can eventually lead to the formation of a neoplasm.

Vaccination-associated tumors that have been described in the literature to have developed in immunization scars as sequelae were mainly melanoma, basal cell carcinoma and squamous cell carcinoma [12]. We need to always keep in mind and actively exclude these mimics if the suspicion of a neoplasm arises when a patient presents with a nonhealing chronic wound.

To date, only 3 cases of DF after smallpox vaccination have been described in the literature $[6,12,13]$. However, none of these were reported to be of the described ulcerating morphology. Notably, other rarer tumors that have been reported to develop after vaccination include melanoma, basal cell carcinoma and squamous cell carcinoma [3, 13]. The development of postvaccination cutaneous neoplasms has not been shown to be associated with any specific type of vaccine being administered, although a significant number reported in the literature occurred secondary to smallpox vaccination $[6,12,13]$; the reason for this remains unclear. 


\section{Vaccination and Granuloma Formation}

We entertained the possibility of a diagnosis of ulcerative aluminum granuloma, a lesion well known to develop in small children after immunization as a hypersensitivity reaction to aluminum adjuvants deposited in the skin upon injection of the vaccine. Cases of such granuloma formation following DTP (diphtheria, tetanus and pertussis) vaccination have been previously documented in the literature $[14,15]$.

The use of aluminum-containing vaccines has been established for many years in the history of immunization. The aluminum adjuvant is thought to be able to prolong the period of adsorption, hence enhancing the stimulatory effect and increasing the immunologic response [14]. The development of granulomatous reactions remains peculiar to these aluminum-adsorbed vaccines.

Histologically, the lesion is usually characterized by foci of necrotizing granulomatous inflammation consisting of lymphoid follicles in the deep dermis and subcutaneous tissues. There is a surrounding dense infiltrate of lympho-histiocytic, eosinophilic and plasma cells. Occasionally, central foci of eosinophilic necrosis can be seen within the inflammatory granuloma, and the eosinophilic crystals stain pink-purple with solochrome cyanine stain [2]. These characteristic histological features were, however, not present in our case, confirming our diagnosis of an atypical DF instead.

\section{Conclusions}

The diagnosis of a common cutaneous neoplasm may not always be straightforward, and in atypical presentations, diagnosis can only be made after careful correlation of the clinical picture with dermoscopic and histopathological findings. Infrequent phenomena, such as ulcerative changes, make clinical diagnosis challenging. We would like to emphasize that common lesions can always present in unusual forms differing from the classical textbook descriptions, and we aim to bring to attention these rare entities. Furthermore, since these morphological variants of DF may mimic malignant neoplasms, judicious clinicopathological correlation and a high index of suspicion regarding unusual-looking lesions is always crucial in order not to miss a possibly aggressive lesion, as consequences can be dire. Although the pathogenesis behind neoplasm development after vaccination still remains unclear, what remains important is the acquisition of sound knowledge and wise judgement to make the right diagnosis to institute correct treatment early.

\section{Statement of Ethics}

The authors have no ethical conflicts to disclose.

\section{Disclosure Statement}

The authors declare no conflicts of interest. 


\section{Case Reports in \\ Dermatology}

\section{References}

1 Hendricks WM: Dermatofibroma occurring in a smallpox vaccination scar. J Am Acad Dermatol 1987;16:146-147.

2 Page EH, Assaad DM: Atrophic dermatofibroma and dermatofibrosarcoma protuberans. J Am Acad Dermatol 1987;17:947-950.

3 Marmelzat WL: Malignant tumors in smallpox vaccination scars: a report of 24 cases. Arch Dermatol 1968;97:400-406.

-4 Fawcett HA, Smith NP: Injection-site granuloma due to aluminum. Arch Dermatol 1984;120:13181322.

-5 Zelger BG, Zelger B: Dermatofibroma (fibrous histiocytoma): an inflammatory or neoplastic disorder? Histopathology 2001;38:379.

-6 Calonje E: Is cutaneous benign fibrous histiocytoma (dermatofibroma) a reactive inflammatory process or a neoplasm? Histopathology 2000;37:278-280.

7 Bandyopadhyay MR, Besra M, Dutta S, Sarkar S: Dermatofibroma: atypical presentations. Indian J Dermatol 2016;61:121.

-8 Sánchez Yus E, Soria L, de Eusebio E, et al: Lichenoid, erosive and ulcerated dermatofibromas. Three additional clinico-pathologic variants. J Cutan Pathol 2000;27:112-117.

-9 Calonje E, Mentzel T, Fletcher CDM: Cellular benign fibrous histiocytoma. Clinicopathologic analysis of 74 cases of a distinctive variant of cutaneous fibrous histiocytoma with frequent recurrence. Am J Surg Pathol 1994;18:668.

10 González-Vilas D, García-Gavín J, Ginarte M, et al: Ulcerated dermatofibroma with osteoclast-like giant cells. J Cutan Pathol 2009;36(suppl 1):16-19.

11 Kiyohara T, Kumakiri M, Kobayashi H, et al: Atrophic dermatofibroma. Elastophagocytosis by the tumor cells. J Cutan Pathol 2000;27:312-315.

12 Waibel KH, Walsh DS: Smallpox vaccination site complications. Int J Dermatol 2006;45:684-688.

13 Bittencourt MJS, Miranda MFR, Parijós AM, et al: Dermatofibroma in a black tattoo: report of a case. An Bras Dermatol 2013;88:614-616.

14 Bordet AL, Michenet P, Cohen C, et al: Post-vaccination granuloma due to aluminium hydroxide (in French). Ann Pathol 2001;21:149-152.

15 Pembroke AC, Marten RH: Unusual cutaneous reactions following diphtheria and tetanus immunization. Clin Exp Dermatol 1979;4:345-348.

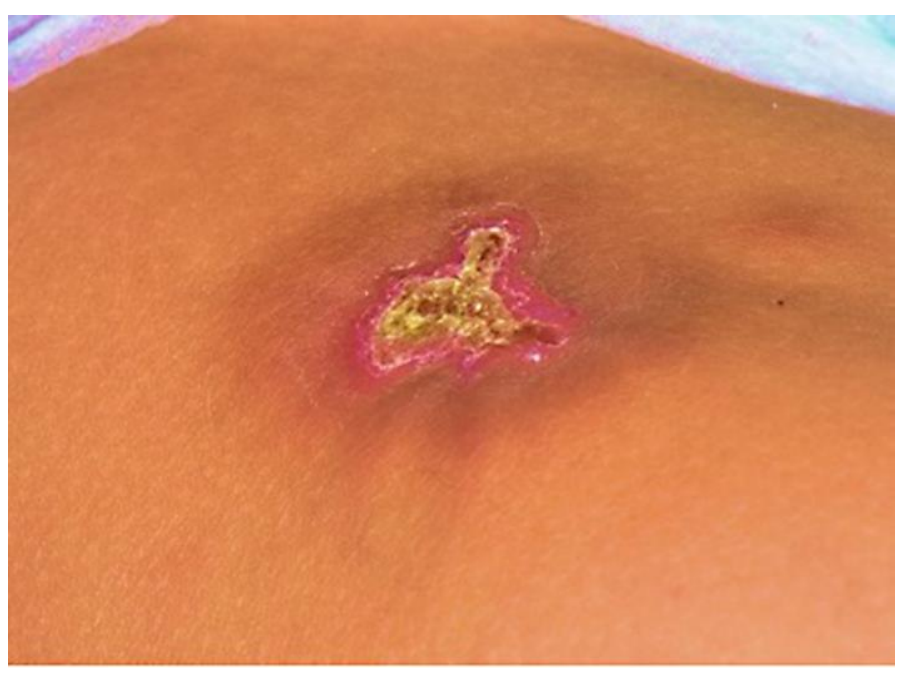

Fig. 1. DF on the right anterolateral thigh with atrophic and ulcerated central scar and a peripheral rim of deeper pigmentation. This is an atypical morphology compared to that of the usual nodular-type characteristics of DFs. 


\section{Case Reports in \\ Dermatology}

Case Rep Dermatol 2016;8:210-217

(C) 2016 The Author(s). Published by S. Karger AG, Basel www.karger.com/cde

Ng et al.: Ulcerated Giant Dermatofibroma following Routine Childhood Vaccination in a Young Boy

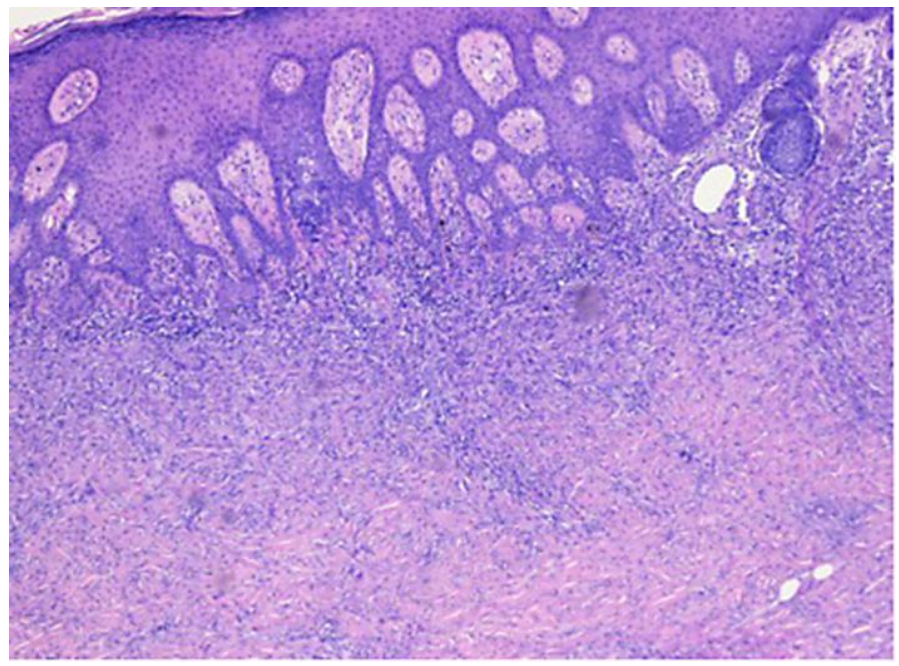

Fig. 2. Epidermal hyperplasia with spindle cell proliferation in the superficial-to-deep dermis under HE stain.

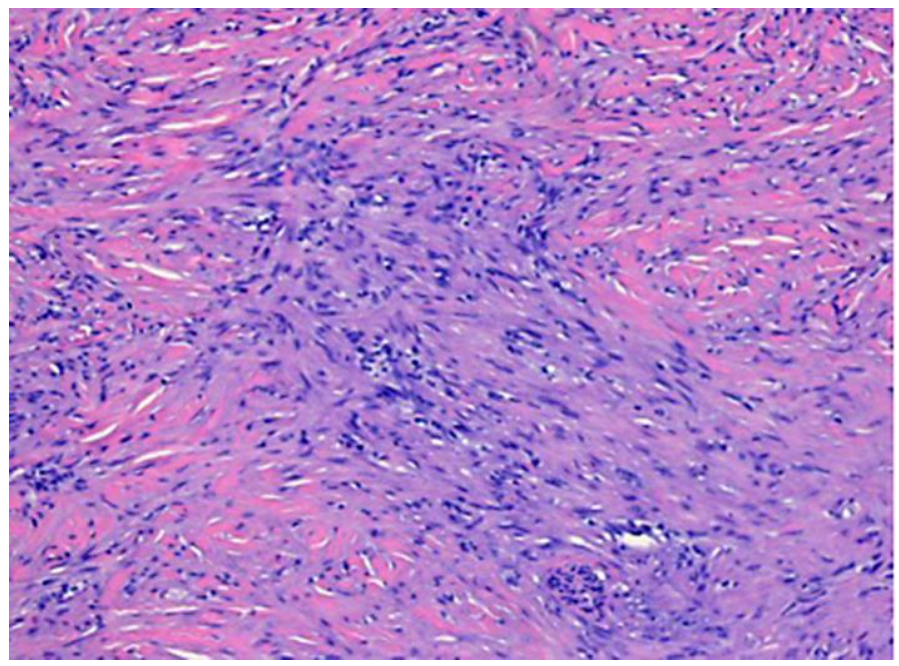

Fig. 3. Spindled fibrohistiocytes arranged in whorls with peripheral collagen trapping. 


\section{Case Reports in \\ Dermatology}

Case Rep Dermatol 2016;8:210-217

(C) 2016 The Author(s). Published by S. Karger AG, Basel www.karger.com/cde

$\mathrm{Ng}$ et al.: Ulcerated Giant Dermatofibroma following Routine Childhood Vaccination in a Young Boy

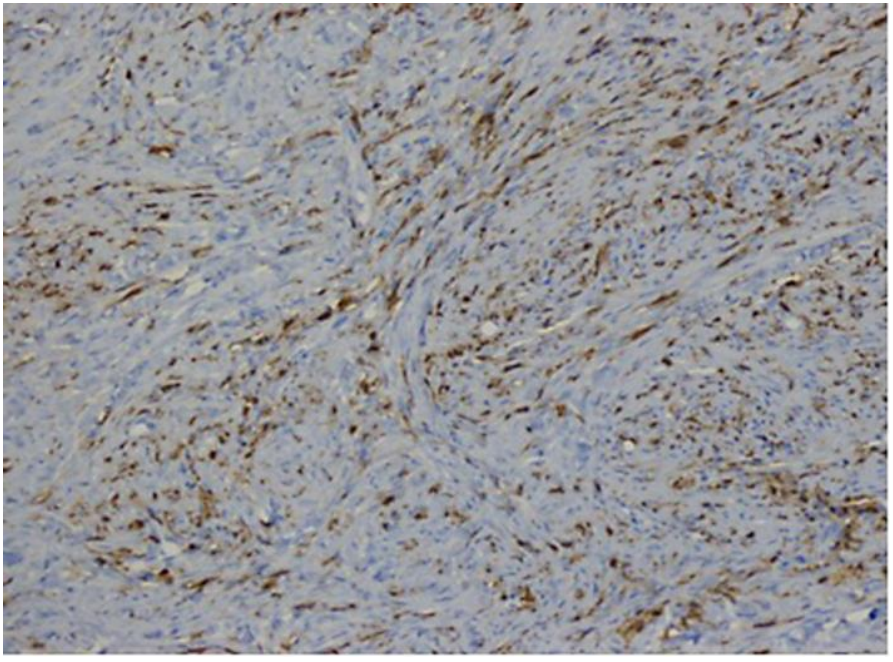

Fig. 4. Spindled cells stained positively for factor XIIIa immunohistochemistry. 